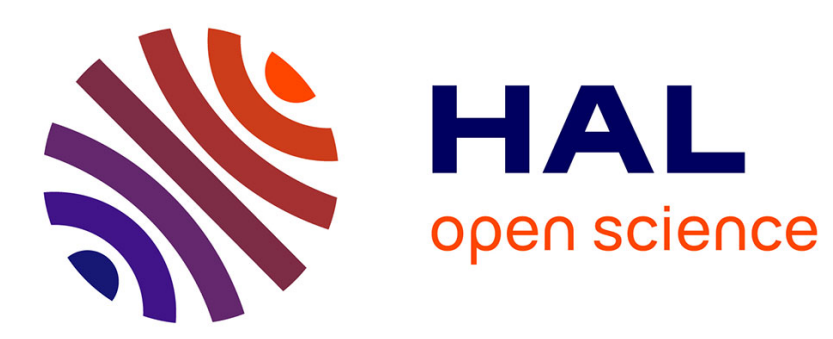

\title{
LPV-Based Autonomous Vehicle Lateral Controllers: A Comparative Analysis
}

Hussam Atoui, Olivier Sename, Vicente Milanés, John Jairo Martinez Molina

\section{To cite this version:}

Hussam Atoui, Olivier Sename, Vicente Milanés, John Jairo Martinez Molina. LPV-Based Autonomous Vehicle Lateral Controllers: A Comparative Analysis. 2021. hal-03197895

\section{HAL Id: hal-03197895 \\ https://hal.science/hal-03197895}

Preprint submitted on 14 Apr 2021

HAL is a multi-disciplinary open access archive for the deposit and dissemination of scientific research documents, whether they are published or not. The documents may come from teaching and research institutions in France or abroad, or from public or private research centers.
L'archive ouverte pluridisciplinaire HAL, est destinée au dépôt et à la diffusion de documents scientifiques de niveau recherche, publiés ou non, émanant des établissements d'enseignement et de recherche français ou étrangers, des laboratoires publics ou privés. 


\title{
LPV-Based Autonomous Vehicle Lateral Controllers: A Comparative Analysis
}

\author{
Hussam Atoui ${ }^{1,2}$, Olivier Sename ${ }^{2}$, Vicente Milanés ${ }^{1}$ and John Jairo Martinez ${ }^{2}$
}

\begin{abstract}
This paper presents a comparative analysis using three Linear Parameter Varying (LPV) control approaches for the lateral control of an autonomous vehicle. Polytopic, gridbased and Linear Fractional Transformation (LFT) lateral controllers are designed, highlighting their benefits and drawbacks from the theoretical design to the real implementation on a real automated Renault $\mathrm{ZOE}$ vehicle. A solution is proposed to deal with both lane change and lane tracking problems, using a single LPV controller, by adapting the look-ahead distance. Each LPV controller is designed based on LPV/ concept. Performance comparison includes computational costs, vehicle performance (i.e. lateral tracking error or control effort optimization) and on-board integration complexity. Simulation and experimental results on a private test track are included to support main findings.
\end{abstract}

\section{INTRODUCTION AND MOTIVATION}

Research on Intelligent Vehicle Highway Systems (IVHS) and Automated Highway Systems (AHS) has been very active in the last decades [1]. Two vehicle control tasks were considered: longitudinal control and lateral control. The former aims mainly to achieve car-following [2] by regulating the speed of the vehicle. The latter minimizes the lateral error by adjusting the steering actuator [3]. Lateral control is used to maintain lane-keeping and lane-changing, i.e. to handle small and large lateral errors.

Different control strategies have been proposed for the lane-keeping task. In [4], a nested PID steering control was formulated for lane-keeping based on a vision system. A fault tolerant control for automated vehicle has been designed in recent researches [5], [6]. A static feedback control was used in [7] combining the longitudinal and lateral controls. In addition, a comparison was done between PID, adaptive, $\mathcal{H}_{\infty}$ and fuzzy logic controllers in [8]. In addition, [9] compares and evaluates the performance of five path-following algorithms according to various disturbances like gust wind, drop of road friction coefficient and inaccurate GPS localization.

Lateral control for overtaking or obstacle avoidance maneuvers has been also tackled in the literature. This concept took a part of the studies concerning the steering rate and the forward distance to be covered by ensuring safety with respect to the vehicles around. Fuzzy logic control [10] and different

*Institute of Engineering Univ. Grenoble Alpes

$1 \mathrm{H}$. Atoui and V. Milanés are with the Research Department, Renault SAS, 1 Avenue de Golf, 78280 Guyancourt, France hussam.atoui, vicente.milanes@renault.com

2 H. Atoui, O. Sename and John J. Martinez are with Univ. Grenoble Alpes, CNRS, Grenoble INP*, GIPSA-lab, 38000 Grenoble, France. olivier.senamedgrenoble-inp.fr, john-jairo.martinez-molina@grenoble-inp.fr optimization techniques as in [11], [12] were used to maintain better scenarios for lane change and collision avoidance.

One of the most used schemes for the lateral control consists of [13], [14]: 1) a feedforward term which generates the desired yaw rate at a varying look-ahead distance with respect to the vehicle's speed; and 2) a feedback compensator that minimizes the current vehicle yaw rate error to reduce the lateral error. In [13], the steering rate is defined to be proportional to both heading and lateral errors, adapting lookahead distance accordingly to vehicle speed. Thus, a Linear Time-Invariant (LTI) controller is designed for a fixed lookahead distance and speed. Each one of those obtained LTI controllers has a different performance to be used in a certain situation. [15] uses this approach to design a switching LTI controller based on Youla-Kucera (YK) parameterization. Two LTI controllers are designed separately having the same speed but different look-ahead distance parameter $d$; the first one with $d=30 \mathrm{~m}$ for managing larger lateral errors smoothly, and the second with $d=15 \mathrm{~m}$ to provide fast tracking capabilities. When the absolute lateral error increases from 0.6 to $3 \mathrm{~m}$, the controller switches its performance from the second to the first. Our paper proposes a solution which solves the same problem, but by adapting the look-ahead distance within the generated yaw rate reference in terms of the absolute lateral error, and using a single robust LPV controller.

Additionally, the LPV concept is used in this work to control the nonlinear lateral dynamics of automated vehicle. Nonlinear control system research field started in the 70s [16]. From a practical point of view, linearization at different operating points was deeply investigated in the literature [17]. LPV concept surged as a control technique that can use LTI synthesis tools to control a nonlinear model. In addition, the studies tended to improve robustness in addition to the optimality which can handle parameter variations in the plant model. If these parameters can be measured online, a gain-scheduling between LTI controllers, designed at different operating points, can be used [18]. Otherwise, the unmeasurable parameters are considered as model uncertainties in the control design to reduce their effects on the closed-loop performance [19].

The importance of the LPV approach to control general nonlinear systems comes from the fact that the system can be written in the form of a quasi-LPV, where the parameter can vary as a function of states, inputs or outputs and not just considered as exogenous inputs. [20] and [21] present successful application example of LPV/robust techniques to different domains. Usually, the formulation of an LPV control problem requires to solve an infinite number of Linear Matrix Inequalities (LMIs) due to the parameter space. Methods were 
proposed to reduce the problem to a finite set of LMIs: polytopic [22], [23], grid-based [24] and LFT [25] approaches that were discussed in the literature. An informative review on the three approaches is drawn in [26].

Even if LPV control has been widely used in different domains, just a few applications can be found in the autonomous driving domain. Specifically:

- The polytopic approach was applied in [27] to design lateral and longitudinal controllers by solving $L Q R / \mathcal{H}_{2}$. Moreover, the lateral control was obtained using an $\mathrm{LPV} / \mathcal{H}_{\infty}$ polytopic controller of the lateral dynamics which varies with respect to the vehicle's speed [28].

- Grid-based approach hasn't been implemented before on lateral control, but it was applied on different systems in the vehicle. For example, authors in [29] controlled the anti-roll bar system by a grid-based LPV/H $\mathcal{H}_{\infty}$ control. Also, it was employed in the electromechanical systems with an application on the electric power steering systems as shown in [30].

- LFT approach hasn't been applied to lateral control previously, it was widely used in the field of aircraft control [31], [32]. In addition, a control design of an underwater vehicle was studied in [33]. Moreover, a diesel engine common injection system was implemented using LFT approach with good results [34].

This paper presents a comparative analysis among the three mentioned LPV approaches to the autonomous vehicle lateral control. The design of the three approaches from a theoretical point of view as well as their application on a real vehicle are presented, including a comparative analysis. Specifically, comparison is carried out attending to the next criteria: 1) controllers are compared by studying their designing procedure which is discussed in a theoretical point of view; 2) it is shown how each controller deals with different chosen situations (noise, disturbance, large lateral errors, low and high speeds, etc...). This is achieved by analysing the lateral errors (to ensure safety) and the control input efforts (to ensure comfortability and actuator limitation) from the simulation and real implementation results; and 3) they are also compared by observing their complexities when applying them on real applications and their effects on the computational costs.

The main contribution of this paper is to develop, experimentally validate and compare three distinct LPV controllers which can deal, comfortably, with both lane change and lane tracking problems. This can be summarized as follows:

- LPV models are structured for the three approaches from the nonlinear bicycle model that vary with respect to the longitudinal velocity.

- To limit the control input effort and to achieve the noise/disturbance rejection performance, LPV $/ \mathcal{H}_{\infty}$ problems are solved using a set of LMIs.

- Input reference is adjusted as a function of the speed and lateral error, modifying the look-ahead parameter accordingly to deal with large lateral errors (lane changing) and the small ones (lane tracking).

- Simulation and experimental results are shown to compare the performance of the controllers concerning track- ing, actuator limitations and noise/disturbance rejection.

\section{MODEL FORMULATION}

The lateral vehicle dynamics is modelled using the wellknown bicycle model [35], [36]. Next subsections describe the plant model and the design of each LPV model.

\section{A. Lateral Bicycle Model}

In [35] and [36], the nonlinear lateral bicycle model is derived as:

$$
\left\{\begin{array}{l}
\dot{v}_{y}=\frac{F_{y f} \cos \delta+F_{y r}}{m}-w v_{x} \\
\dot{w}=\frac{F_{y f} l_{f} \cos \delta-F_{y r} l_{r}}{I},
\end{array}\right.
$$

where $v_{x}, v_{y}$ and $w$ are the longitudinal, lateral and rotational velocities in the vehicle's frame, respectively. $\delta$ is the control input, the steering front angle of the front tire. $I, m, l_{f}$ and $l_{r}$ are the vehicle's inertia, mass and the distance from the center of gravity to the front and rear wheel axes respectively. $F_{y f}$ and $F_{y r}$ are the lateral forces applied to the front and rear tires, respectively:

$$
\begin{gathered}
F_{y f}=C_{f}\left(\delta-\tan ^{-1}\left(\frac{v_{y}}{v_{x}}+\frac{l_{f} w}{v_{x}}\right)\right), \\
F_{y r}=C_{r} \tan ^{-1}\left(-\frac{v_{y}}{v_{x}}+\frac{l_{r} w}{v_{x}}\right),
\end{gathered}
$$

For simplicity, the lateral forces are linearized by considering small slip angles. Then, the lateral forces are approximated as follows:

$$
\begin{gathered}
F_{y f}=C_{f}\left(\delta-\frac{v_{y}}{v_{x}}+\frac{l_{f} w}{v_{x}}\right), \\
F_{y r}=C_{r}\left(-\frac{v_{y}}{v_{x}}+\frac{l_{r} w}{v_{x}}\right),
\end{gathered}
$$

where $C_{f}$ and $C_{r}$ are the stiffness of the front and rear wheel-tires respectively. Notice that the linear bicycle model with constraint lateral acceleration has been stated as a good approximation of the nonlinear model [37]. Then this model could be sufficient for daily passenger vehicles.

\section{B. LPV Model Structures}

After choosing $\rho(t)=v_{x} \in \mathbb{R}^{n_{\rho}}$ as a varying parameter, and assuming small steering front angles $(\sin (\delta) \approx \delta$ ), and small slip angles, the LPV state-space representation is written as:

$$
G(\rho)\left\{\begin{array}{c}
\dot{x}(t)=A(\rho) x(t)+B u(t) \\
y(t)=C x(t)+D u(t)
\end{array}\right.
$$

where:

$$
\begin{aligned}
& x(t)=\left[\begin{array}{l}
v_{y} \\
w
\end{array}\right] \in \mathbb{R}^{n_{x}}, u(t)=\delta,, y(t)=w, B=\left[\begin{array}{c}
\frac{1}{m} C_{f} \\
\frac{1}{I} C_{f} l_{f}
\end{array}\right], \\
& A(\rho)=\left[\begin{array}{cc}
-\frac{C_{r}+C_{f}}{m v_{x}} & -\frac{l_{f} C_{f}-l_{r} C_{r}}{m v_{x}}-v_{x} \\
-\frac{l_{f} C_{f}-l_{r} C_{r}}{I v_{x}} & -\frac{l_{f}^{2} C_{f}+l_{r}^{2} C_{r}}{I v_{x}}
\end{array}\right] .
\end{aligned}
$$

The parameter-dependency of the LPV model differs from one approach to another [21]. Specifically, the control synthesis of the polytopic approach requires an affine parameterdependency. However, the gridding approach does not require such assumption. Finally, the LFT model is defined to be a lower or upper Linear Fractional Representation (LFR) between a known LTI model and a varying-parameter block. The following discussion shows how each approach is formulated. 


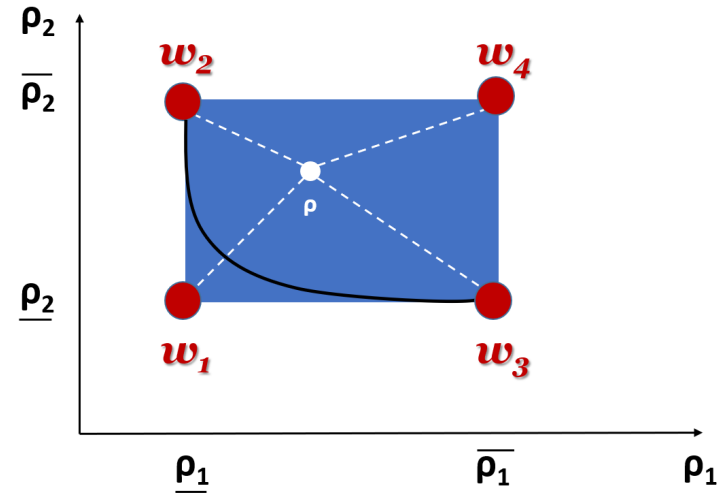

Fig. 1: The polytope

\section{1) Polytopic Model}

The polytopic model is defined in a convex hull bounded by the parameters extremums (see Fig. 1). It is formulated as a convex combination between the vertices of the polytope. Two conditions must be satisfied: 1) the input and output matrices should be independent of the varying parameters, this is usually solved by pre-filtering the input or output; and 2) the model must be affine with respect to the varying parameters. So, (5) is written as:

$$
A_{p o l}(\rho)=\left[\begin{array}{cc}
-\frac{C_{r}+C_{f}}{m} \rho_{2} & -\frac{C_{f} l_{f}-C_{r} l_{r}}{m} \rho_{2}-\rho_{1} \\
-\frac{C_{f} l_{f}-l_{r} C_{r}}{I} \rho_{2} & -\frac{C_{f} l_{f}^{2}+l_{r}^{2} C_{r}}{I} \rho_{2}
\end{array}\right],
$$

where $\rho_{1}$ and $\rho_{2}$ are $v_{x}$ and $\frac{1}{v_{x}}$ respectively. Figure 1 shows the polytope formed by the extremums of the two parameters. Notice that the parameter vector is represented as a convex combination between the 4 vertices, where $\rho=\sum_{i=1}^{2^{n} p} \mu_{i} \omega_{i}$ and $\sum_{i=1}^{2^{n} p} \mu_{i}=1, \mu_{i} \geq 0 \forall i$ being

$$
\begin{array}{ll}
\omega_{1}=\left(\underline{\rho_{1}}, \underline{\rho_{2}}\right), & \mu_{1}=\frac{\left|\overline{\rho_{1}}-\rho_{1}\right|}{\left|\overline{\rho_{1}}-\underline{\rho_{1}}\right|} \times \frac{\left|\overline{\rho_{2}}-\rho_{2}\right|}{\left|\overline{\rho_{2}}-\underline{\rho_{2}}\right|} \\
\omega_{2}=\left(\underline{\rho_{1}}, \overline{\rho_{2}}\right), & \mu_{2}=\frac{\left|\overline{\rho_{1}}-\rho_{1}\right|}{\left|\overline{\rho_{1}}-\underline{\rho_{1}}\right|} \times \frac{\left|\underline{\rho_{2}}-\rho_{2}\right|}{\left|\overline{\rho_{2}}-\underline{\rho_{2}}\right|} \\
\omega_{3}=\left(\overline{\rho_{1}}, \underline{\rho_{2}}\right), & \mu_{3}=\frac{\left|\overline{\rho_{1}}-\rho_{1}\right|}{\left|\overline{\overline{\rho_{1}}}-\underline{\rho_{1}}\right|} \times \frac{\left|\overline{\rho_{2}}-\rho_{2}\right|}{\left|\overline{\rho_{2}}-\underline{\rho_{2}}\right|} \\
\omega_{4}=\left(\overline{\rho_{1}}, \overline{\rho_{2}}\right), & \mu_{4}=\frac{\left|\underline{\rho_{1}}-\rho_{1}\right|}{\left|\overline{\rho_{1}}-\underline{\rho_{1}}\right|} \times \frac{\left|\underline{\rho_{2}}-\rho_{2}\right|}{\left|\overline{\overline{\rho_{2}}}-\underline{\rho_{2}}\right|}
\end{array}
$$

The LPV model is then written as a convex combination of the LTI systems obtained at the vertices of the polytope:

$$
\left[\begin{array}{c|c}
A_{\text {pol }}(\rho) & B_{\text {pol }}(\rho) \\
\hline C_{\text {pol }}(\rho) & D_{\text {pol }}(\rho)
\end{array}\right]=\sum_{i=1}^{2^{n_{p}}} \mu_{i}(\rho)\left[\begin{array}{l|l}
A\left(\omega_{i}\right) & B\left(\omega_{i}\right) \\
\hline C\left(\omega_{i}\right) & D\left(\omega_{i}\right)
\end{array}\right]
$$

where $n_{p}=2$ is the number of the varying parameters.

2) Grid-based Model

The grid-based LPV model is a model interpolated over a set of LTI models that are linearized at different operating points. This approach can be considered when the parameter

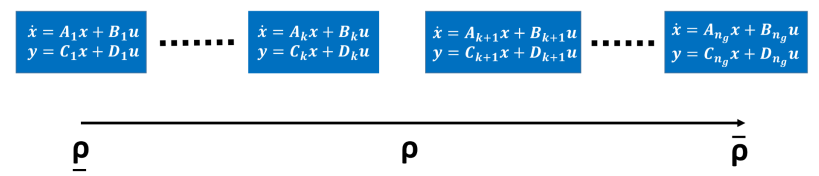

Fig. 2: The gridded matrices

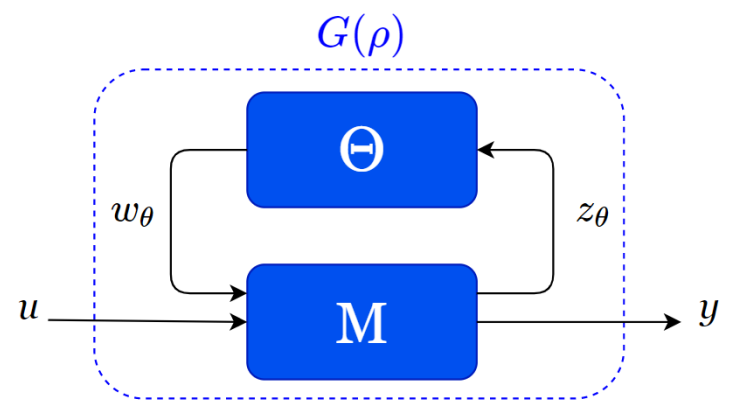

Fig. 3: Linear fractional representation of a parameter-varying system

dependency of the model is nonlinear (without model reformulation) [26], [24]. So, the only needed parameter here is $\rho=v_{x}$. The varying parameter is gridded to a chosen number of $n_{g}$ grid points as shown in Fig. 2.

The gridding approach uses any kind of interpolation (linear or nonlinear) between the gridded models to compute the LPV model. Suppose that, at an instant, the longitudinal velocity $\rho \in\left[\rho_{k}, \rho_{k+1}\right] \mathrm{m} / \mathrm{s}$, the linear interpolation of the state-space matrices:

$$
\left[\begin{array}{c|c}
A_{\text {grid }}(\rho) & B_{\text {grid }}(\rho) \\
\hline C_{\text {grid }}(\rho) & D_{\text {grid }}(\rho)
\end{array}\right]=\sum_{i=k}^{k+1} \alpha_{i}(\rho)\left[\begin{array}{c|c}
A_{i} & B_{i} \\
\hline C_{i} & D_{i}
\end{array}\right],
$$

where,

$$
\alpha_{k}=\frac{\rho_{k+1}-\rho}{\rho_{k+1}-\rho_{k}} \quad \text { and } \quad \alpha_{k+1}=\frac{\rho-\rho_{k}}{\rho_{k+1}-\rho_{k}}
$$

\section{3) LFT model}

The LFT approach defines the model as the upper LFR between a known LTI model and a parameter block as shown in Fig. 3 [25]. At each instant, the parameters in the block $\Theta$ are updated being the input of the LTI model M. In general, the upper LFT interconnection of the shown model is written as:

$$
\begin{gathered}
{\left[\begin{array}{c}
\dot{x} \\
z_{\theta} \\
y
\end{array}\right]=\left[\begin{array}{ccc}
A & B_{\theta} & B \\
C_{\theta} & D_{\theta \theta} & D_{\theta 1} \\
C & D_{1 \theta} & D
\end{array}\right]\left[\begin{array}{c}
x \\
w_{\theta} \\
u
\end{array}\right]} \\
w_{\theta}=\Theta z_{\theta}
\end{gathered}
$$

where $\Theta$ is the time-varying operator block introduced as:

$$
\Theta=\operatorname{blockdiag}\left(\theta_{1} I_{r_{1}}, \ldots, \theta_{k} I_{r_{k}}\right)
$$

being $r_{i}>1$ which presents the number of occurrences of the varying-parameter $\theta_{i}$. Notice that $\theta_{i}$ can be normalized to be always $\in[-1,1]$, which makes $M$ represents the nominal model when $\theta_{i}=0 \forall i$. 
Let us consider the system model (4), considering the parameter $v_{x}$ varying as:

$$
v_{x}=v_{x_{0}}+a \theta
$$

where $v_{x_{0}}=\frac{v_{x_{\text {min }}}+v_{x_{\max }}}{2}$ represents the nominal value of $v_{x}$, and $a=\frac{v_{x_{\max }}-v_{x_{\min }}}{2}$ represents the rate of variation when $\theta$ varies in $[-1,1]$. Then, (4) can be rewritten under the LFT form (11) where:

$$
\begin{aligned}
& A=\left[\begin{array}{cc}
-\frac{C_{r}+C_{f}}{m v_{x_{0}}} & -\frac{l_{f} C_{f}-l_{r} C_{r}}{m v_{x_{0}}}-v_{x_{0}} \\
-\frac{l_{f} C_{f}-l_{r} C_{r}}{I v_{x_{0}}} & -\frac{l_{f}^{2} C_{f}+l_{r}^{2} C_{r}}{I v_{x_{0}}}
\end{array}\right] \\
& B_{\theta}=\left[\begin{array}{cccc}
-\frac{a}{v_{x_{0}}} & 0 & -\frac{a^{2}}{v_{x_{0}}} & 0 \\
0 & 0 & 0 & -\frac{a}{v_{x_{0}}}
\end{array}\right] \\
& C_{\theta}=\left[\begin{array}{cc}
-\frac{C_{r}+C_{f}}{m v_{x_{0}}} & -\frac{l_{f} C_{f}-l_{r} C_{r}}{m v_{x_{0}}}+v_{x_{0}} \\
0 & 1 \\
0 & 0 \\
-\frac{l_{f} C_{f}-l_{r} C_{r}}{I v_{x_{0}}} & -\frac{l_{f}^{2} C_{f}+l_{r}^{2} C_{r}}{I v_{x_{0}}}
\end{array}\right] \\
& D_{\theta \theta}=\left[\begin{array}{cccc}
-\frac{a}{v_{x_{0}}} & 0 & -\frac{a^{2}}{v_{x_{0}}} & 0 \\
0 & 0 & 0 & 0 \\
0 & 1 & 0 & 0 \\
0 & 0 & 0 & -\frac{a}{v_{x_{0}}}
\end{array}\right] \\
& D_{\theta 1}=0_{4 \times 1}, D_{1 \theta}=0_{1 \times 4}, \Theta=\theta \times I_{4}
\end{aligned}
$$

And finally, the state-space matrices of $G(\rho)$ are scheduled as:

$$
\begin{gathered}
A_{L F T}(\rho)=A+B_{\theta} \Delta_{\theta} C_{\theta} \\
B_{L F T}(\rho)=B+B_{\theta} \Delta_{\theta} D_{\theta 1} \\
C_{L F T}(\rho)=C+D_{1 \theta} \Delta_{\theta} C_{\theta} \\
D_{L F T}(\rho)=D+D_{1 \theta} \Delta_{\theta} D_{\theta 1} \\
\Delta_{\theta}=\Theta\left(I-D_{\theta \theta} \Theta\right)^{-1}
\end{gathered}
$$

It is worth noting that, in the LFT approach, the LPV model is converted as in Fig. 3 in a single LTI model feeded by a parameter dependent input. It differs from the polytopic and grid-based approach where a set of LTI models is handled.

\section{LPV $/ \mathcal{H}_{\infty}$ CONTROL DESIGN}

Fig 4 presents the control block diagram where an LTI actuator model $G_{a c t}$ (first order transfer function + input delay) is added to the bicycle model dynamics $\left(\delta_{c}\right.$ is the controller output). Note that the input delay is described as a first order transfer function, so as a result, $G_{a c t}$ is a second order transfer function. Considering this structure, the controller $\mathbf{K}(\rho)$ is designed for the three approaches using $\mathrm{LPV} / \mathcal{H}_{\infty}$ concept. Notice that $w_{\text {ref }}$ represents the yaw rate reference.

Control performance criteria in $\mathcal{H}_{\infty}$ control theory are given by frequency domain functions. Two weighting functions $W_{e}$ and $W_{u}$ are used to achieve tracking and actuator limitations performances respectively. The objective is to achieve both performances with a trade-off between minimizing the lateral error and ensuring the driving comfort.

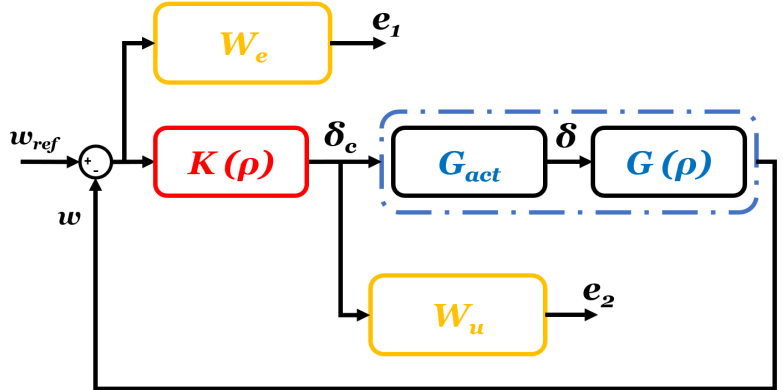

Fig. 4: Control design scheme

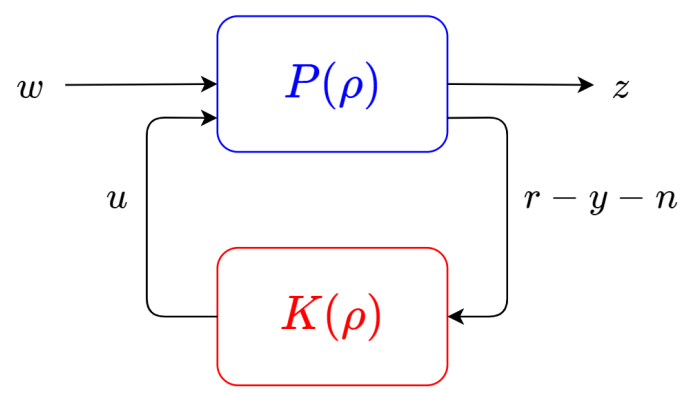

Fig. 5: General control configuration

\section{A. Tracking specification $\left(W_{e}\right)$}

The weighting transfer function is chosen as:

$$
W_{e}(s)=\frac{\frac{s}{M_{s}}+w_{b}}{s+w_{b} \epsilon}
$$

where the parameters $M_{s}, w_{b}$ and $\epsilon$ are tuned as follows:

- $M_{s}=2$, to ensure robustness at any frequency.

- $w_{b} \geq 10$, to choose the speed of rising time-response.

- $\epsilon \leq 10^{-4}$, to represent the steady-state tracking error.

\section{B. Specification on the control input limitations $\left(W_{u}\right)$}

A filter is used to minimize the effort of the steering actuator control $\delta_{c}$. This filter is designed as:

$$
W_{u}(s)=\frac{s+\frac{w_{b_{u}}}{M_{u}}}{\epsilon_{u} s+w_{b_{u}}}
$$

The parameters $M_{u}, w_{b_{u}}$ and $\epsilon_{u}$ are adopted as:

- $M_{u}$ represents the limitations on the maximum allowed effort of the actuators.

- $w_{b_{u}}$, is related to the actuator bandwidth.

- $\epsilon_{u} \leq 10^{-2}$, is concerned with the noise rejection from the control inputs at high frequencies.

\section{Generalized Plant}

Using the plant model and the weighting functions, Fig. 4 is converted to build a general control configuration as in Fig. 5 . The generalized plant $P(\rho)$ is structured to consider the chosen weights in addition to the LPV model $\left(G(\rho) \times G_{a c t}\right)$. Thus the state vector of $P(\rho)$ is $x_{P}=\left[\begin{array}{llll}x & x_{a c t} & x_{W_{e}} & x_{W_{u}}\end{array}\right]^{T}$, and the controlled output $z=\left[\begin{array}{ll}e_{1} & e_{2}\end{array}\right]^{T}$ represents the objective function to be optimized when designing the controller. $w=$ 


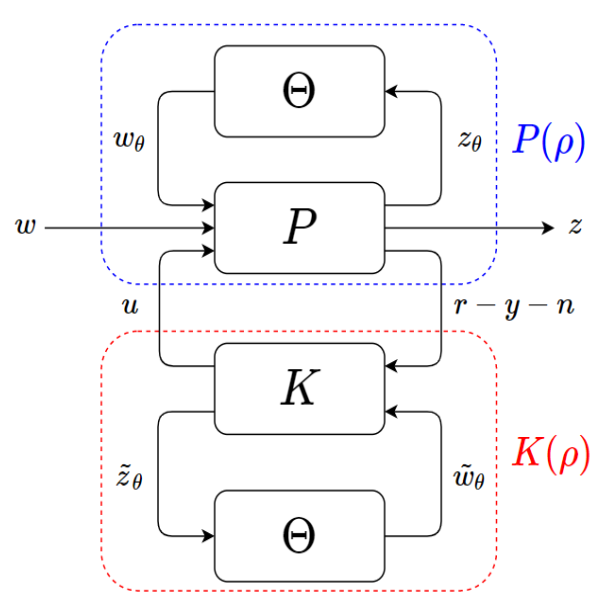

Fig. 6: LFT general control configuration

$\left[\begin{array}{lll}r & d & n\end{array}\right]^{T}$ is the exogenous input, where $r, d$ and $n$ are the desired reference, input disturbance and the measurable noises respectively.

For the polytopic and the gridding approaches, the statespace representation of $P(\rho)$ (see Fig. 5) has the form:

$\left[\begin{array}{c}\dot{x}_{P} \\ e_{1} \\ e_{2} \\ r-y-n\end{array}\right]=\left[\begin{array}{c|cccc}A_{P}(\rho) & B_{1} & B_{2} & B_{3} & B_{4} \\ \hline 0 & W_{e} & 0 & -W_{e} & -W_{e} G(\rho) \\ 0 & 0 & 0 & 0 & W_{u} \\ 0 & 1 & 0 & -1 & -G(\rho)\end{array}\right]\left[\begin{array}{c}x_{P} \\ r \\ d \\ n\end{array}\right]$

In the polytopic approach, there exist four generalized plants, each one is related to a corresponding vertex of the polytope. However, a set of $n_{g} P_{i}$ 's are formulated along the gridded points for the gridding approach.

Fig. 6 shows the generalized plant $P(\rho)$ as an upper LFR between a parameter-invariant $P$ and the parameter block $\Theta$ for designing an LPV/LFT controller. Using this approach, $P$ is then structured as:

$$
\left[\begin{array}{c}
\dot{x}_{P} \\
z_{\theta} \\
e_{1} \\
e_{2} \\
r-y-n
\end{array}\right]=\left[\begin{array}{c|ccccc}
A_{P} & B_{\Theta} & B_{1} & B_{2} & B_{3} & B_{4} \\
\hline C_{\theta} & D_{\theta \theta} & D_{\theta 1} & D_{\theta 2} & D_{\theta 3} & D_{\theta 4} \\
0 & 0 & W_{e} & 0 & -W_{e} & -W_{e} G(\rho) \\
0 & 0 & 0 & 0 & 0 & W_{u} \\
0 & 0 & 1 & 0 & -1 & -G(\rho)
\end{array}\right]\left[\begin{array}{c}
x_{P} \\
w_{\Theta} \\
{\left[\begin{array}{c}
r \\
d \\
n
\end{array}\right]} \\
u
\end{array}\right]
$$

\section{D. $L P V / \mathcal{H}_{\infty}$ Control Synthesis}

For the rest of the paper, the longitudinal speed is considered to be bounded as:

$$
v_{x} \in[3,30] \mathrm{m} / \mathrm{s}
$$

The main objective of the $\mathcal{H}_{\infty}$ control is to minimize the $\mathcal{L}_{2}$ induced gain from the external input $w$ to the controlled output $\mathrm{z}=\left[\begin{array}{ll}e_{1} & e_{2}\end{array}\right]^{T}$. This is achieved by solving the following $\mathcal{L}_{2}$ induced minimization problem:

$$
\|z\|_{2} \leq \gamma_{\infty}\|w\|_{2}
$$

and $\gamma_{\infty}>0$, to be minimized, represents how much the demanded performance is achieved. If $\gamma_{\infty}<1$, the demanded performance is totally achieved by the controller. Among the three approaches, each controller is designed by solving its corresponding LMI-based optimisation problem. A complete overview of the synthesis and implementation complexity of LPV approaches can be found in [38]. For each approach, the complexity of LPV controller existence conditions is determined in terms of the size of LMIs and the number of decision variables. Note that the obtained controller $K(\rho)$ is an LPV Dynamic Output Feedback Controller.

\section{1) Polytopic Approach}

Fig. 1 shows that the polytopic approach is considering the parameters $v_{x}$ and $\frac{1}{v_{x}}$ as two independent parameters. However, they depend on each other as a function of the black curve (i.e. $y=1 / x$ ). Since the polytope is a very large set compared to the real parameter variation rule (and mainly due to $w_{4}$ ), this may lead to conservatism when solving the optimisation problem on the 4 vertices. Thus, a solution to this problem is drawn in [39], where the number of vertices is reduced from $2^{n_{p}}$ to $n_{p}+1$. So, the polytope in Fig. 1 can be reduced to $\mathcal{C}_{\mathcal{O}}\left\{w_{1}, w_{2}, w_{3}\right\}$, and then, the coefficients $\mu_{i}^{\prime} s$ will be:

$\mu_{1}=1-\left(\mu_{2}+\mu_{3}\right), \quad \mu_{2}=\frac{\left|\rho_{2}-\rho_{2}\right|}{\overline{\mid \rho_{2}}-\underline{\rho_{2}} \mid}, \quad \mu_{3}=\frac{\left|\rho_{1}-\rho_{1}\right|}{\overline{\overline{\rho_{1}}}-\underline{\rho_{1}} \mid}$

In the polytopic approach, the problem is solved in the framework of quadratic stability of the closed-loop system. This is obtained by solving a set of LMIs at the vertices of the polytope with a constant Lyapunov function [22]. The existence conditions of this approach show the number of LMIs to grow by $\mathcal{O}\left(2^{n_{p}}\right)$. Also, the number of decision variables is computed to be $n_{x}\left(n_{x}+1\right)$ [38]. As a result, a controller $K_{i}$ is obtained at each vertex and $K(\rho)$ is computed as (in the case of non-reduced polytope and $\forall \rho$ inside the polytope of Fig. 1):

$$
\left[\begin{array}{c|c}
A_{K}(\rho) & B_{K}(\rho) \\
\hline C_{K}(\rho) & D_{K}(\rho)
\end{array}\right]=\sum_{i=1}^{2^{n_{p}}} \mu_{i}(\rho)\left[\begin{array}{c|c}
A_{K_{i}} & B_{K_{i}} \\
\hline C_{K_{i}} & D_{K_{i}}
\end{array}\right]
$$

\section{2) Grid-based Approach}

Grid-based approach formulates the problem in the context of robust stability [40] by using a parameter-dependent Lyapunov function along the gridded axis [24]. Notice that a basis function is chosen to write the Lyapunov function in terms of the varying parameters. An example on the lateral vehicle control problem, the Lyapunov function $X(\rho)$ is chosen to be linearly dependent (order 1 ) on the varying parameter $\rho=v_{x}$ :

$$
X(\rho)=X_{0}+\rho X_{1},
$$

where $X_{0}$ and $X_{1}$ are unknown constant matrices to be computed from the LMIs. From this point of view, one can see an advantage in the optimisation problem of this approach where it reduces the conservatism of the polytopic approach. Notice that the LMIs include the absolute maximum ratechange of the parameter. Studying the existence conditions of this control approach, the number of LMIs grows with $\mathcal{O}\left(n_{g}^{n_{\rho}}\right)$. To determine the number of decision variables, let us assume that the parameter-dependent Lyapunov matrix is parameterized as:

$$
X(\rho)=X_{0}+\sum_{i=q}^{s} a_{i}(\rho) X_{i},
$$


then the number of decision variables is $\frac{1}{2} n_{x}\left(n_{x}+1\right)\left(n_{\rho}^{X}+\right.$ +1 ), where $n_{\rho}^{X}=s-q+1$. For more details, the LMIs are derived in Theorem 4.3.1 in [24]. As a result, a set of controllers is obtained where each one corresponds to a frozen value in the gridded axis of the parameter. When $\rho \in\left[\rho_{k}, \rho_{k+1}\right]$, the controller $K(\rho)$ can be linearly interpolated as [26]:

$$
\left[\begin{array}{c|c}
A_{K}(\rho) & B_{K}(\rho) \\
\hline C_{K}(\rho) & D_{K}(\rho)
\end{array}\right]=\sum_{i=k}^{k+1} \alpha_{i}(\rho)\left[\begin{array}{c|c}
A_{K_{i}} & B_{K_{i}} \\
\hline C_{K_{i}} & D_{K_{i}}
\end{array}\right],
$$

where,

$$
\alpha_{k}=\frac{\rho_{k+1}-\rho}{\rho_{k+1}-\rho_{k}} \quad \text { and } \quad \alpha_{k+1}=\frac{\rho-\rho_{k}}{\rho_{k+1}-\rho_{k}}
$$

\section{3) LFT Approach}

The LPV/LFT controller synthesis mainly relies on the $\mathcal{S}$ procedure [41]. The solution of the LMI-based optimization problem is detailed in [25], where continuous and discretetime LMI problems are discussed. The LMI size is typically smaller than that in the polytopic approach since there is no anymore a set of LMIs to be solved for a set of parameter values (see Fig. 2 in [26] for more details). However, a trade-off is found where the number of decision variables of LFT is much bigger than the polytopic approach when $n_{\rho}$ increases $\left(n_{x}\left(n_{x}+1\right)+2 n_{\Theta}\left(2 n_{\Theta}+1\right)\right.$ where $\left.\Theta \in \mathbb{R}^{n_{\Theta} \times n_{\Theta}}\right)$. Control synthesis is formulated in two steps: 1) A quadruple $\left(R, S, L_{3}, J_{3}\right)$ is found - by solving 2 LMIs - to ensure the solvability of the control problem (check Theorem 5.1 in [25]); and 2) The gain-scheduling controller is then computed using the quadruple and solving another LMI with one decision variable containing the controller matrices (Algorithm 6.1 in [25]). Then, the designed controller is written in the form of a lower LFR $F_{l}(K, \Theta)$, and obtained as follows:

$$
K(\rho)=F_{l}(K, \Theta)=K_{11}+K_{12} \Theta\left(I-K_{22} \Theta\right)^{-1} K_{21}
$$

being $K(\rho)$ defined as:

$$
\left[\begin{array}{c}
u \\
\tilde{z}_{\theta}
\end{array}\right]=\left[\begin{array}{l|l}
K_{11} & K_{12} \\
\hline K_{21} & K_{22}
\end{array}\right]\left[\begin{array}{c}
r-y-n \\
\tilde{w}_{\theta}
\end{array}\right]
$$

where $K_{11}, K_{12}, K_{21}$ and $K_{22}$ are obtained from the LMI's. Thus, the state-space matrices of $K(\rho)$ are computed as:

$$
\begin{gathered}
A_{K}(\rho)=A_{K}+B_{K_{\theta}} \Delta_{\theta} C_{K_{\theta}} \\
B_{K}(\rho)=B_{K}+B_{K_{\theta}} \Delta_{\theta} D_{K_{\theta 1}} \\
C_{K}(\rho)=C_{K}+D_{K_{1 \theta}} \Delta_{\theta} C_{K_{\theta}} \\
D_{K}(\rho)=D_{K}+D_{K_{1 \theta}} \Delta_{\theta} D_{K_{\theta 1}} \\
\Delta_{\theta}=\Theta\left(I-D_{K_{\theta \theta}} \Theta\right)^{-1}
\end{gathered}
$$

\section{E. Frequency Domain Analysis}

This subsection analyses the designed controllers in frequency-domain in order to check if the requirements are satisfied by the controllers. This is achieved using several sensitivity functions compared to the weights designed previously. Additionally, an LTI controller designed at the fixed-nominal speed $v_{x}=14 \mathrm{~m} / \mathrm{s}$ is added to the analysis to show the benefits of using parameter-varying approaches.

To observe the tracking performance, the error sensitivity function $S$ is drawn as shown in Fig. 7. Sensitivity functions of the tracking error to the reference are drawn with

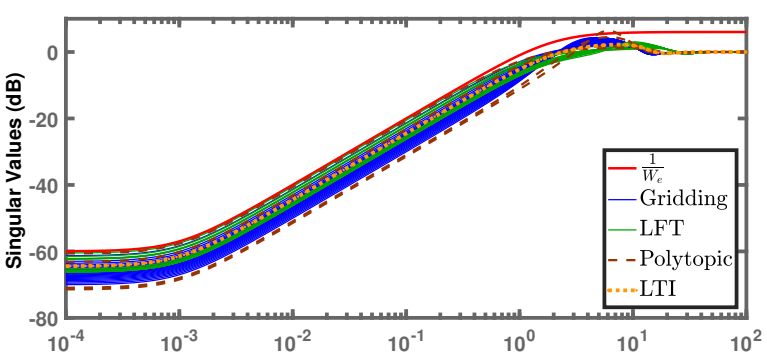

Fig. 7: Sensitivity Functions $S=\frac{w_{r e f}-w}{w_{r e f}}$

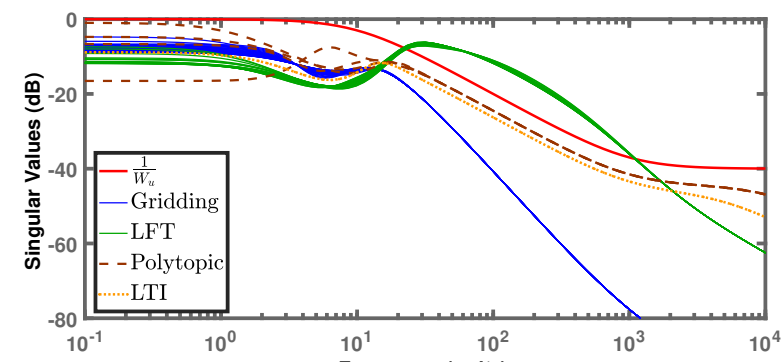

Fig. 8: Sensitivity Functions $K S=\frac{\delta}{w_{r e f}}$

the required performance $\frac{1}{W_{e}}$. Notice that each of the LPV approaches has several sensitivity functions where each one is referred to a corresponding value of speed (in gridding and LFT approaches) or a corresponding vertex (in polytopic approach). At low frequencies, all the controllers achieve the demanded steady-state tracking error. Also, all the controllers are respecting the requirements at high frequencies related to robust margin $\left(\max _{\forall w}\left\|\frac{r-y}{r}\right\|<6 d B\right)$.

On the other hand, the analysis of the control input is carried out to evaluate the sensitivity to the noise. To do so, the control sensitivity function $K S$ of each controller is computed. Fig. 8 shows the sensitivity functions of the control input $\delta$ with respect to the reference, and the requirements on the actuator limitations $\frac{1}{W_{u}}$. It is shown that all the controllers are respecting the demanded limitations with a small exceeding in the bandwidth of the LFT approach. One can appreciate how the gridding approach shows the best noise rejection at high frequencies compared to the others.

\section{SIMULATION RESULTS}

All the simulations are employed in MATLAB using the nonlinear model (1)-(2) designed for a Renault ZOE vehicle. The simulations are performed in discrete-time domain with a sampling time $T_{s}=10 \mathrm{~ms}$. The proposed control strategies are implemented in simulation following the scheme in Fig. 9. The look-ahead system block is added to perform smoothly both lane-keeping and lane-changing (i.e. small and large lateral errors respectively). It modifies the yaw rate reference when having large lateral errors (initial error in autonomous starting mode, sudden lane-changes, etc.). Since the desired curvature is inversely proportional to the look-ahead distance [13], a smooth or aggressive steering can be obtained by 


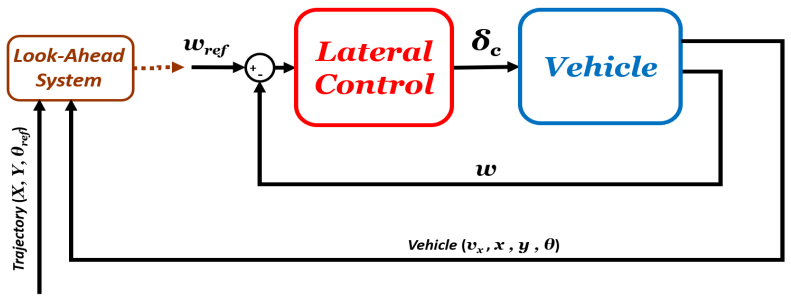

Fig. 9: Control implementation scheme

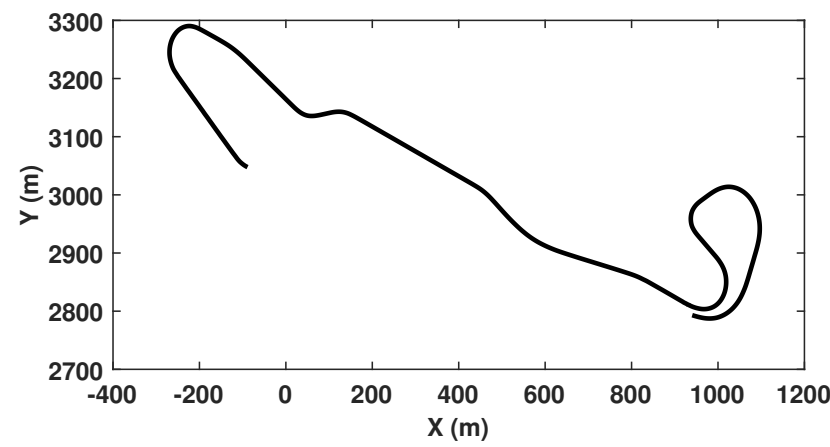

Fig. 10: Reference Trajectory

changing the look-ahead distance. Specifically, as much as the lateral error $y_{e}$ increases, the look-ahead distance $d$ is increased linearly as a function of the lateral error as follows:

$d\left(v_{x}, y e\right)=\operatorname{sat}\left(\frac{d_{\text {nom }}\left(v_{x}\right)}{2} \times\left(1+a b s\left(y_{e}\right)\right),\left[d_{\text {nom }}\left(v_{x}\right), d_{\text {max }}\right]\right)$

where $d_{n o m}\left(v_{x}\right)$ is the nominal look-ahead distance tuned at each speed $v_{x}$ for small lateral errors $\left(y_{e} \leq 1 m\right), d_{\max }$ is the maximum saturation of look-ahead distance chosen when $y_{e} \geq 3 \mathrm{~m} . d_{\max }$ is chosen depending on the facing situation where it should ensure that the vehicle is not losing forward information (we choose it here as $d_{\max }=40 \mathrm{~m}$ ).

As discussed in subsection III-D, the LPV approaches have different ways of implementation and interpolation. Each one differs with respect to its complexity and time consuming, which makes it worthy to see how they can be simplified and what will be the effects of such simplifications. Due to the limited number of real tests which can be done, this section discusses different ways of implementations for each approach

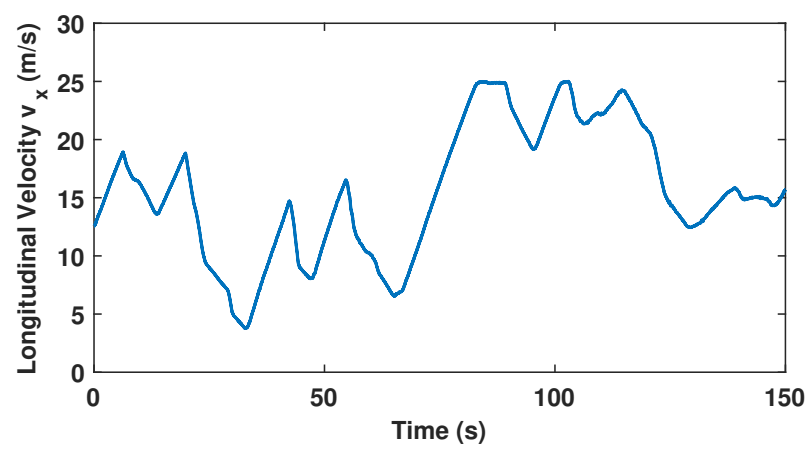

Fig. 11: Speed profile measured from a real test $(\mathrm{m} / \mathrm{s})$ separately and their impact on the system performance. The trajectory in Fig. 10 is used to test the lateral control performance. Vehicle speed input is recovered from a real test (see Fig. 11). Some critical situations were chosen to show the functionality of the look-ahead system and to compare the response of the different designed controllers, where:

- a large lateral error is presented at the initialization time,

- the reference positioning system $(X, Y)$ is recovered from a real test with noises.

- the rate of the varying parameter $v_{x}$ is increased to be large enough at some times $(t \in[70,80] \mathrm{s})$,

- a high speed $v_{x}=25 \mathrm{~m} / \mathrm{s}$ is reached at time $t=80 \mathrm{~s}$,

- two successive aggressive maneuvers at high speed (around $23 \mathrm{~m} / \mathrm{s}$ ) are carried out between $t=105 \mathrm{~s}$ and $t=115 s$.

\section{A. LPV Approaches Limitations}

This subsection describes the main limitations of each approach from the implementation point of view.

\section{1) Polytopic Approach}

As mentioned in paragraph III-D1, the polytope is reduced to decrease its problem conservatism. Thus, to analyse the benefit of this reduction, two polytopic LPV controllers (one with four vertices and the other with three vertices) are designed. Both of them are simulated separately and the results of the obtained lateral errors and steering input angles are represented in Fig. 12 and 13.

Table I, Fig. 12 and 13 show that both controllers achieve approximately the same lateral error and steering performance. This result is due to the fact that the bicycle model is not highly nonlinear in terms of the speed $v_{x}$. Moreover, Fig. 12 and 13 show that the initial lateral error is minimized smoothly, thanks to the look-ahead system which adapts the look-ahead distance as the lateral error increases (see Fig. 14). Notice that Fig. 14 shows only the first few seconds since the look-ahead distance is no more adapted for $t>6 s$, i.e. $d=d_{\text {nom }}\left(v_{x}\right) \forall t>6 s$ since the lateral error does not exceed $0.6 \mathrm{~m}$.

Finally, Table I shows the lateral error RMS for both polytopic controllers. One can appreciate the benefits of reducing the polytope, especially when having varying-parameters that depend on each other.

TABLE I: RMS of the lateral error using the polytopic approaches

\begin{tabular}{|c|c|c|}
\hline & 4-Vertices Polytope & Reduced Polytope \\
\hline RMS & 0.98 & 1.01 \\
\hline
\end{tabular}

\section{2) Gridding Approach}

The gridding approach reduces the problem of conservatism by using a parameter-varying Lyapunov function. Gridded controllers are often interpolated either linearly or nonlinearly to obtain the current-state controller. The benefit of this interpolation is that even if the parameter goes out of its extremum bounds that was considered in the control design (i.e. $v_{x}<3 \mathrm{~m} / \mathrm{s}$ ), the controller still can be interpolated with negative coefficients. Designing a gridded-based LPV controller, the gridded axis of the varying parameter should 


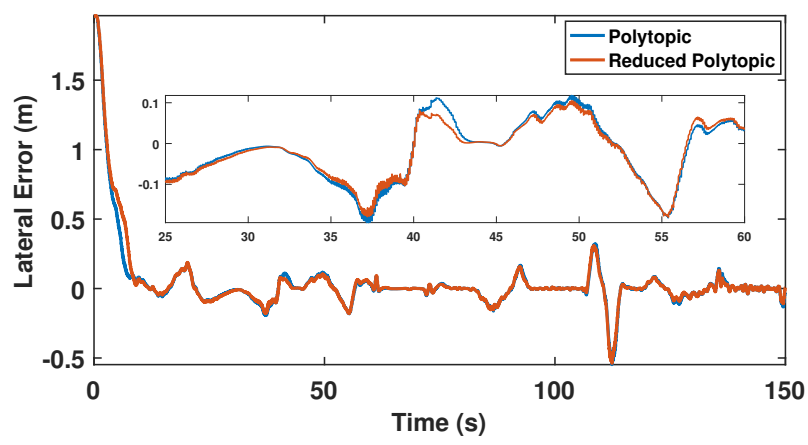

Fig. 12: Lateral error using the polytopic approaches $(m)$

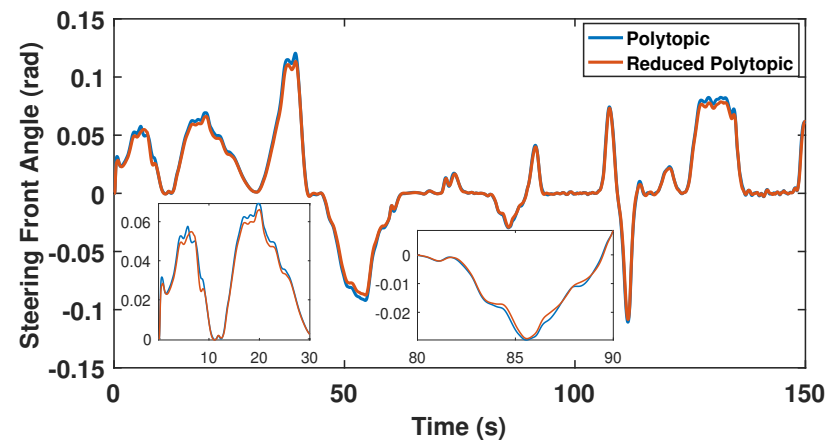

Fig. 13: Steering front angle $\delta$ using the polytopic approaches $(\mathrm{rad})$

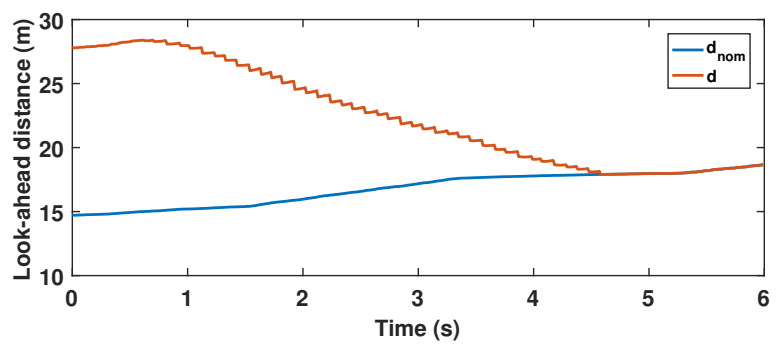

Fig. 14: Look-ahead distance $d(\mathrm{~m})$

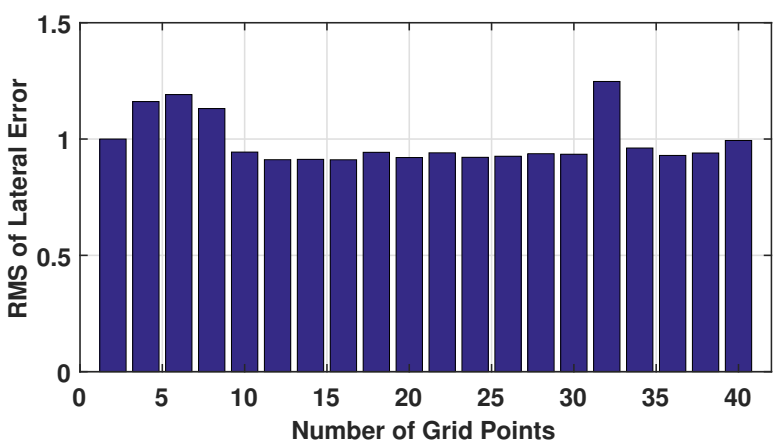

Fig. 15: Normalized RMS of the lateral error with respect to different number of gridding points $n_{g}$ be drawn. This axis can be gridded starting from two gridpoints until infinity. Thus, it is worthy to study the effect of increasing the number of gridding points on the performance of the system.

A set of tests is done at each chosen number of gridding points and the RMSs of the lateral errors are computed. The chosen number of gridding points are the even numbers from 2 to 40 grid-points $(2,4, \ldots, 38,40)$. Fig. 15 shows the normalised RMS of the lateral errors from each test. Notice that all of them are normalized with respect to the test of $n_{g}=2$ to make it easier for comparison, being the first test RMS equals to 0.263 . It is clearly shown that increasing the number of gridding points may not always improve the performance.

Thus, it can be concluded that, in the used model, the performance of the gridded controller is not significantly affected by the number of gridding points.

3) LFT Approach

The implementation of the LFT controller (29) is simpler where it consists of a state-space control system which varies with an input parameter $\Delta_{\theta}$, unlike the previous approaches that have a set of controllers to be interpolated between each other. However, an online inverse must be done at each sample time. This may lead to a limitation during simulation or specifically in real implementations. Thus, a method has been used in [34] to avoid online calculations. A look-up table of the inverted matrix is computed at each chosen grid-point along the parameter range, and then, an interpolation is done during simulation.

The controller with online inverse, the one with interpolation and the nominal controller (at $v_{x}=17.5 \mathrm{~m} / \mathrm{s}$ ) are tested (see Fig. 16 and 17 for details). Table II presents the RMS lateral error, where there is no significant difference between the tracking performances of the tested controllers. Fig. 16 shows that the performance of the interpolated controller (yellow line) differs a bit from the one with online inverse (red line) when the parameter variation rate increases (i.e. $t \in[40,60] \mathrm{s}$ ). On the other hand, it can be clearly observed that when the speed approaches the nominal speed $\left(v_{x}=17.5 \mathrm{~m} / \mathrm{s}\right)$, the nominal LFT controller (blue line) acts more closely to the one with online inverse (red line) $(t \in[135,140] s)$. Notice that at high speeds $(t \in[90,100])$, the nominal LFT controller is showing a small difference in the lateral error, whereas the other two controllers are having exactly the same performance regarding also the steering action (see Fig. 17). As a result, the interpolated LFT controller showed a similar performance as the actual one which may help in reducing the computational cost in real implementations.

TABLE II: RMS of the lateral error using the LFT approaches

\begin{tabular}{|c|c|c|c|}
\hline & Nominal LFT & LFT with online inverse & Interpolated LFT \\
\hline RMS & 0.1849 & 0.1683 & 0.17 \\
\hline
\end{tabular}

\section{B. LPV Approaches Robustness Test}

The most promising result of each LPV approach is retained and tested again with the LTI one (at $v_{x}=17.5 \mathrm{~m} / \mathrm{s}$ ) by modifying some vehicle parameters (vehicle mass and tire stiffness). The mass of the vehicle changes by the number 


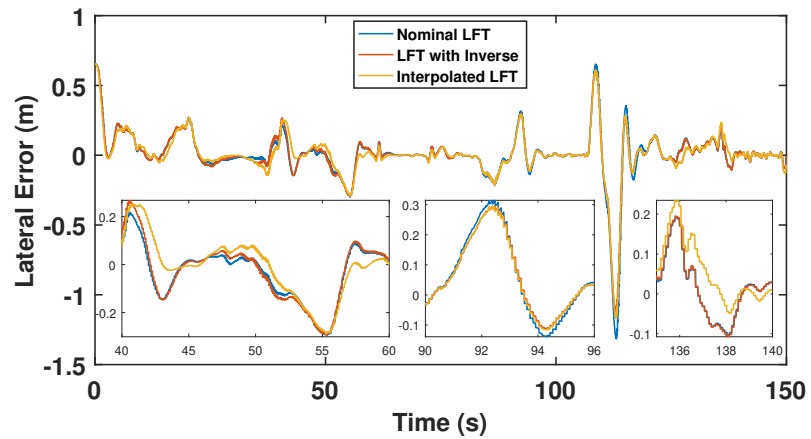

Fig. 16: Lateral error using the LFT approaches $(m)$

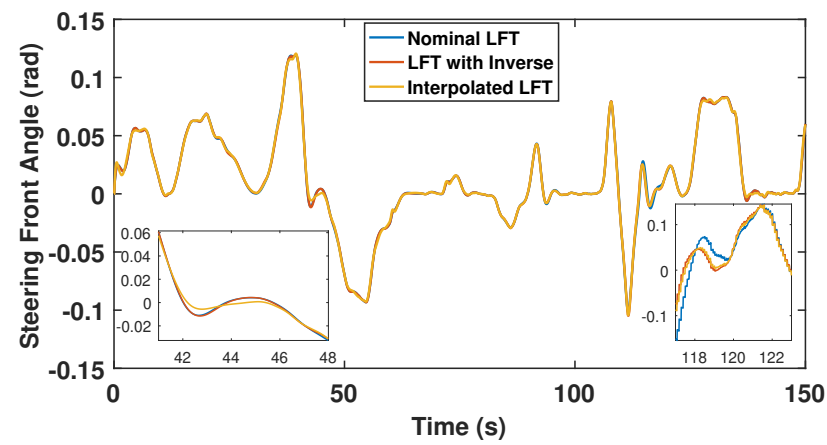

Fig. 17: Steering front angle $\delta$ using the LFT approaches $(\mathrm{rad})$

of passengers. Moreover, the tire stiffness is indirect to be estimated (see [42] for instance), and it varies by time when the tire loses its quality. Then, the following test is important to study the robustness of the designed controllers in terms of such uncertainties. In this test, the mass of the vehicle is chosen arbitrarily to be increased by $400 \mathrm{Kg}$ and each front and rear tire stiffness is adjusted by $30 \%$.

The obtained results are shown in Fig. 18 and 19. Again, one can appreciate the success of the look-ahead system which helps in minimizing the initial lateral error smoothly, i.e. low steering actuation (see Fig. 19). Fig. 18 and Table III show that the tested controllers achieve comparable minimization of the lateral error, with the best tracking performance achieved by the gridded controller. The interpolated LFT seems to have the lowest robustness where it maintains the highest RMS lateral error with more steering overshoots as observed in Fig. 19. The steering rate RMS of the reduced polytopic controller shows the highest value in Table III, and it has the highest overshoot when performing the initial lateral error (Fig. 19). Finally, all the compared controllers are considered to be robust to the injected uncertainties thanks to the used $\mathcal{H}_{\infty}$ concept.

TABLE III: RMS of the lateral error for robustness comparison

\begin{tabular}{|c|c|c|c|c|}
\hline & LTI & Reduced Polytopic & $\begin{array}{c}\text { Gridding } \\
\left(\mathrm{n}_{g}=16\right)\end{array}$ & Interpolated LFT \\
\hline RMS & 0.257 & 0.266 & 0.251 & 0.33 \\
\hline
\end{tabular}

\section{REAL IMPLEMENTATION}

The four last controllers compared in simulation are then tested on a robotized electric Renault ZOE shown in Fig. 20.

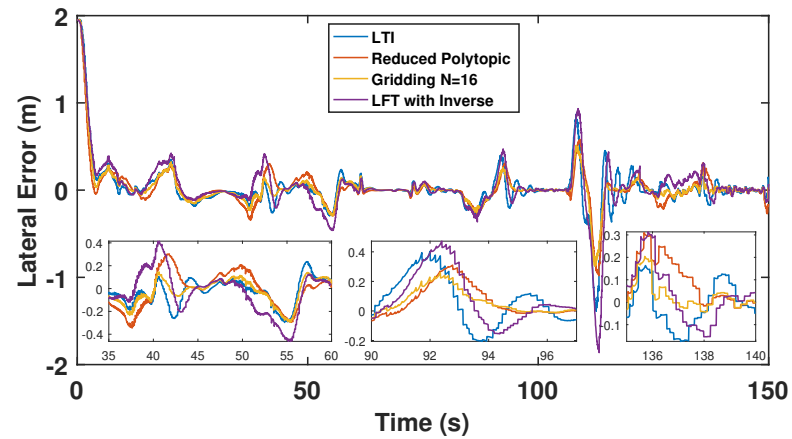

Fig. 18: Lateral error for robustness comparison (m)

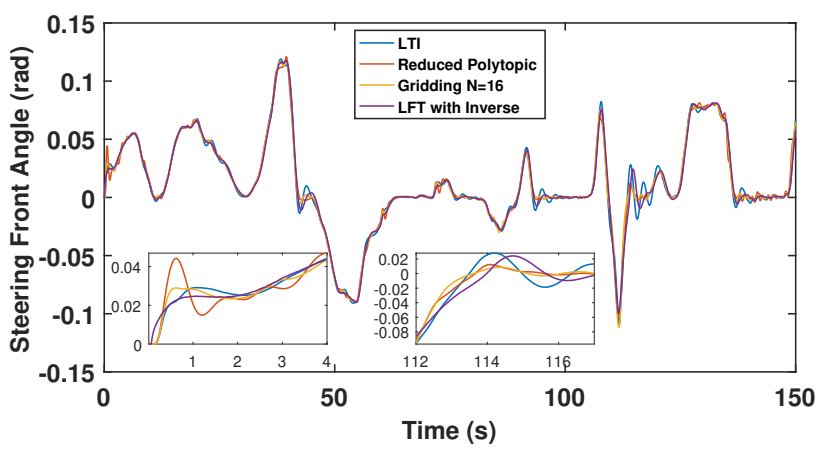

Fig. 19: Steering front angle for robustness comparison (m)

This automated vehicle is adapted for lateral and longitudinal controls by computer-controlled steering and pedal actuators. Vehicle speed and the global coordinates are measurable using GPS and IMU. As previously stated, actuator dynamics are modelled using an LTI model which influence uncertain dynamics between the real plant and the designed model. The test results of the designed controllers are discussed concerning their implementation, the acceptability of the resulted performance and the actuator limitations.

The tests are done in the closed track Satory shown in Fig. 21. This track contains bad road conditions and roadinclinations which allows to evaluate the controller robustness. The first part of the test describes the response of the controllers at high speeds. The second part concerns the precision of lateral control at optimal speeds chosen coherently depending on the road curvature. Fig. 22 shows the longitudinal

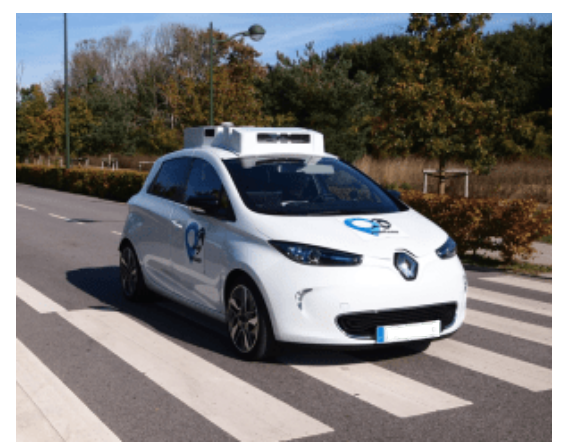

Fig. 20: Renault ZOE automated vehicle 
TABLE IV: RMS of the steering front rate for robustness comparison

\begin{tabular}{|c|c|c|c|c|}
\hline & LTI & Reduced Polytopic & $\begin{array}{c}\text { Gridding } \\
\left(\mathrm{n}_{g}=16\right)\end{array}$ & Interpolated LFT \\
\hline RMS & 0.017 & 0.018 & 0.017 & 0.017 \\
\hline
\end{tabular}

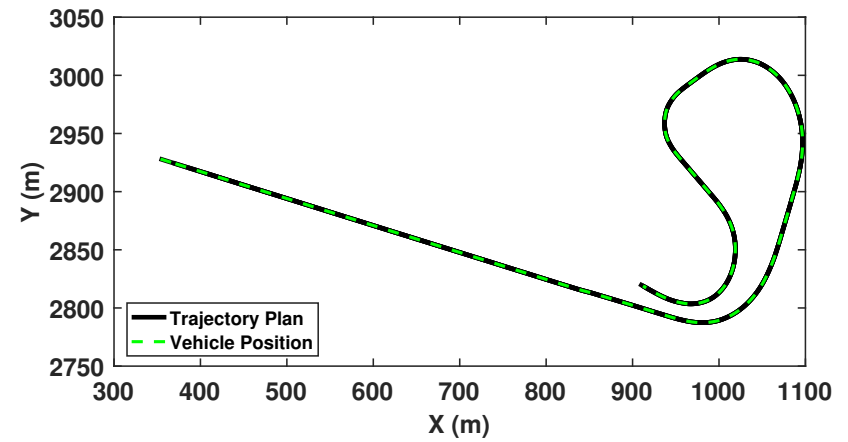

Fig. 21: Satory trajectory plan and vehicle position

speed evolution over time which is considered as an external parameter of the LPV mode. For the coming analysis, the limitations of the used steering actuator are as follows:

$$
|\dot{\delta}|_{\max }=0.2,|\delta|_{\max }=0.4,
$$

Figure 23 and Table V show that all the controllers succeed to minimize the lateral error, however Fig. 24 and 25 show that the control input effort is quiet different. One can observe that both the polytopic and the LTI controllers are sensitive to noises, especially at high speeds (when $t \leq 60 \mathrm{~s}$ ). This can be justified by the conservatism problem of the polytopic approach, and the highly uncertain dynamics reached at high speeds worsen the LTI performance. In fact, this uncertainty is not caused only by the bicycle model, but by the actuator model. Since $G_{a c t}$ is used as an LTI system where actually it varies with speed and quiet changes at high speeds. Then, reaching speeds around $13 \mathrm{~m} / \mathrm{s}$ and below, both controllers performs better with lower sensitivity to noises. On the other hand, the LFT and the gridding controllers show better performance in all situations with a little difference in the minimization of the lateral error. Regarding the steering front angle in Fig. 24, these two controllers have the same evolution with less noises even at high speeds.

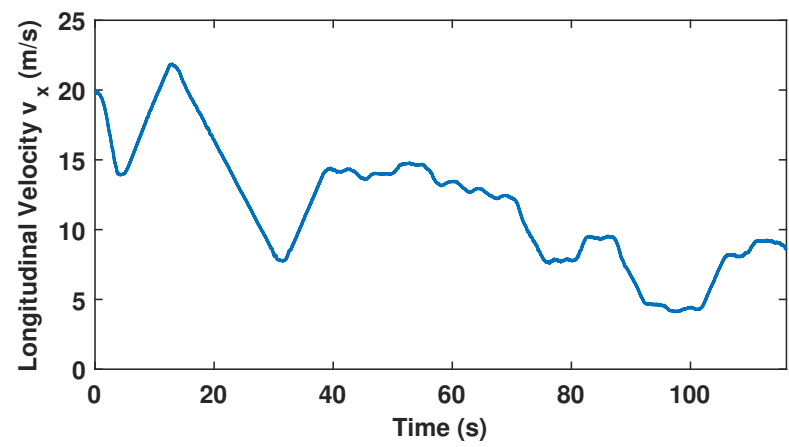

Fig. 22: Experimental longitudinal speed $(\mathrm{m} / \mathrm{s})$

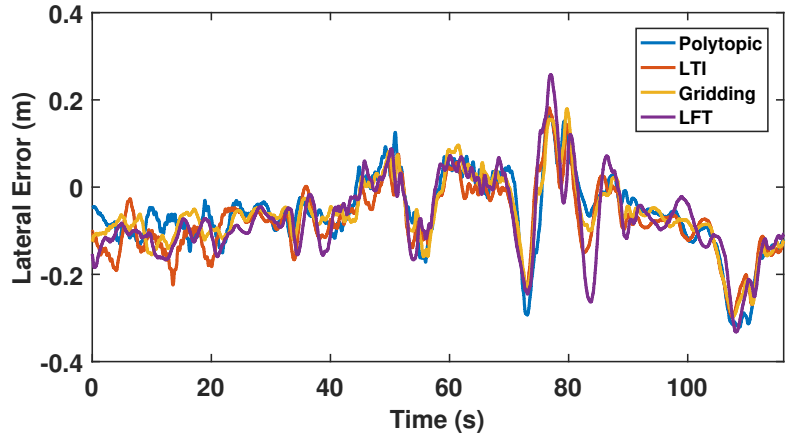

Fig. 23: Experimental lateral error of the LTI and LPV controllers $(m)$

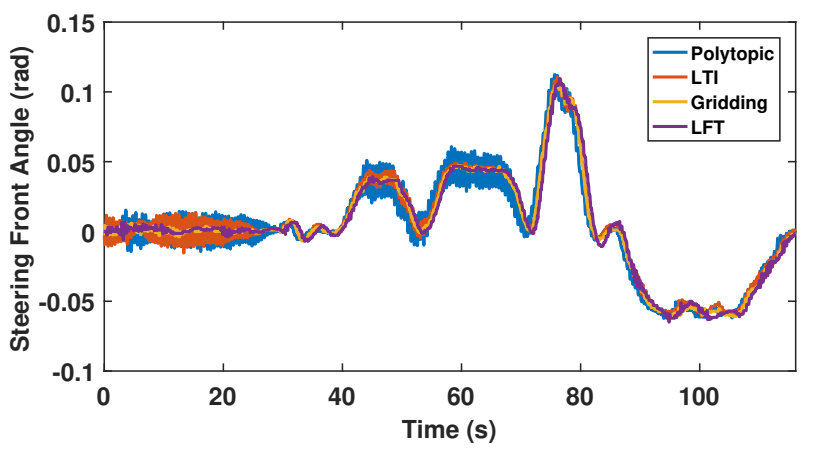

Fig. 24: Experimental steering front angle of the LTI and LPV controllers $(\mathrm{rad})$

Fig. 25 shows the steering rate input for the tested controllers. Again the LFT and the gridding controllers are less noisy than the one obtained by the LTI and the polytopic controllers. Table VI presents that the gridding approach shows the less control effort. Notice that these results support the frequency domain analysis in paragraph III-E, especially the analysis of noise sensitivity at high frequencies.

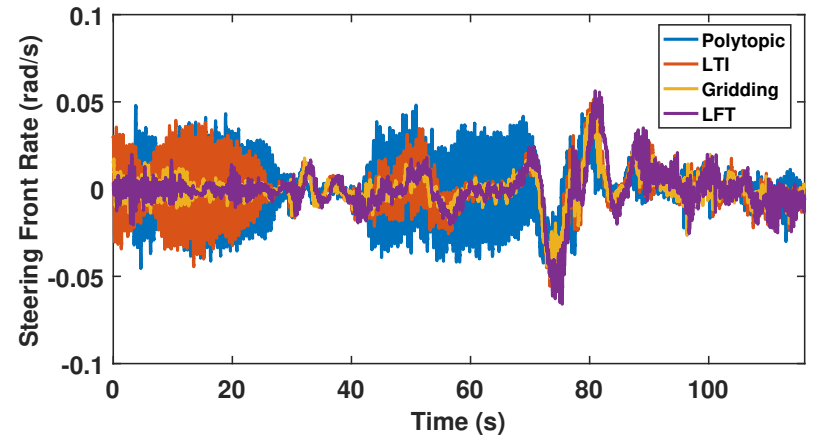

Fig. 25: Experimental steering front rate of the LTI and LPV controllers $(\mathrm{rad} / \mathrm{s})$

TABLE V: RMS of the lateral error for experimental comparison

\begin{tabular}{|c|c|c|c|c|}
\hline & Polytopic & LTI & Gridding & LFT \\
\hline RMS & 0.1473 & 0.1105 & 0.1025 & 0.1096 \\
\hline
\end{tabular}


TABLE VI: RMS of the steering front rate for experimental comparison

\begin{tabular}{|c|c|c|c|c|}
\hline & Polytopic & LTI & Gridding & LFT \\
\hline RMS & 0.0263 & 0.0149 & 0.0107 & 0.0129 \\
\hline
\end{tabular}

\section{SUMMARY OF COMPARISON}

This section presents a general review on the comparison done between the three approaches under the main proposed criteria. It is divided into two main parts, each one discusses the main collected comparisons introduced above:

\section{A. About The Three LPV Approaches}

\section{1) $L P V$ Formulations}

In section II, the nonlinear model was reformulated using the LPV approaches. It has shown that the type of parameterdependency of the model affects the shape and the complexity of the LPV state-space representation.

For the polytopic approach, the system should be written in a way to be affine with respect to the varying parameters, and the input and output matrices should be constant. This may increase the number of parameters which increases the conservatism of the optimisation problem. Regarding the LFT model in (14), a varying parameter leaded a 4-dimensional parameter block $\Theta$ by the cause of its number of occurrences. This explains a real problem facing the applications of LFT approach. Some research studies are done on how to reduce the LFT state-space model [43], especially by the researchers working on aircraft control. Finally, the gridding approach showed a simplicity in model formulation where a set of state-space matrices are obtained at a chosen number of operating points, regardless of the parameter-dependency and its occurrences.

2) $L P V / \mathcal{H}_{\infty}$ Control Design

Section III introduced the $\mathcal{H}_{\infty}$ control design applied to the studied LPV approaches. It has stated the complexity of the existence conditions of each approach in terms of its LMI size and the number of decision variables. Regarding those LMIs, the polytopic approach has the simplest optimisation problem where the known concept of LTI/H $\mathcal{H}_{\infty}$ is applied at the vertices of the polytope with a constant Lyapunov function. However, its LMI size grows exponentially when $n_{p}$ increases, $\mathcal{O}\left(2^{n_{p}}\right)$. This leads to conservatism which is decreased by reducing some vertices [39], but still can not be totally erased. The LFT approach comes to decrease the size of LMIs in polytopic approach, but it requires bigger number of decision variables [26]. The problem of conservatism can be solved using a parameter-dependent Lyapunov function which exists in the grid-based approach. The LMI size in this approach grows by $\mathcal{O}_{g}^{n_{\rho}}$, so it is much bigger than that in the polytopic and LFT approaches when having $n_{g}>2$. Also, the complexity of the grid-based approach appears significantly when increasing the order of the parameter-dependent Lyapunov function $\left(n_{\rho}^{X}\right)$.

\section{B. Experimental Part}

This part plays a vital role in choosing the suitable controller of real applications, where there are many limitations in terms of computer power and costs.

\section{1) Simulation Results}

Section IV has presented the performance of each control approach with its implementation limitation. The main concluding remarks obtained are:

- The reduction of the polytope decreases the conservatism of the optimisation problem, and thus obtaining better performance.

- Using the gridding approach, the system performance is independent from the number of chosen grid-points.

- The LFT controller could be interpolated instead of its gain-scheduling (29) obtaining similar performance.

2) Real Implementation Procedure

Starting with the way of implementation of each approach, the polytopic controller is known to be interpolated as a convex combination of the designed LTI controllers at the polytopic vertices. Thus, those LTI controllers must be saved in a look-up table and then interpolated at each sampling time. It is similar with the gridding approach, but the set of gridded controllers will be linearly interpolated. However, the scheduling of the LFT approach is different, where the matrix $\Delta_{\theta}$ contains a matrix which must be inverted online at each sample time, and this increases the computational time. Since the interpolated LFT shows a performance close to the gainscheduling, it can be implemented using linear interpolation as the gridding approach.

\section{3) Experimental Results}

The last figures show how the studied controllers deal with different situations. For example, the LTI and the polytopic controllers are more sensitive to noises at high speeds, i.e. when the system dynamics change significantly. However, the LFT and the gridding controllers are more robust to handle this kind of uncertainty when the approximated model used in the control design differs from the actual system.

As a result, although the grid-based approach needs to solve more LMIs for more decision variables compared to the polytopic one. However, it provides general model formulation for any parameter-dependency, and it is has the lowest conservatism among the three discussed approaches. On the other hand, the gain-scheduling structure of the LFT approach seems to be the most complex, and its LMIs contain huge number of decision variables compared to the other two. Thus, the grid-based approach could be proposed as a powerful and performant for LPV control applications.

\section{CONCLUSIONS}

This paper has proposed a theoretical and experimental comparison of the LPV approaches for the lateral control of autonomous vehicle. The gridded-based model has the simplest structure with less conservatism in optimisation among the others. The weighting parameters used in control design can physically translate the real actuator limitations to a filter added to the optimisation problem.

The practical limitations of each approach was discussed by observing the simulation results which were obtained from different chosen critical scenarios. Then the results obtained in real implementation showed interesting results regarding the minimization of the lateral error, which encourages the 
TABLE VII: Overview of discussed approaches

\begin{tabular}{|c|c|c|c|c|c|c|}
\hline Controller & $\begin{array}{l}\text { Parameter } \\
\text { dependency }\end{array}$ & $\begin{array}{l}\text { Conservatism } \\
\text { with large } n_{p}\end{array}$ & $\begin{array}{l}\text { LMI size/ } \\
\text { memory growth }\end{array}$ & Number of decision variables & Simulation results & $\begin{array}{l}\text { Experimental } \\
\text { results }\end{array}$ \\
\hline Polytopic & Affine & High & $\mathcal{O}\left(2^{2}\right)$ & 6 & $\begin{array}{l}\text { Polytopic reduction leads } \\
\text { better performance }\end{array}$ & $\begin{array}{l}\text { High sensitivity } \\
\text { to noises }\end{array}$ \\
\hline LFT & Rational & Medium & $\mathcal{O}(2)$ & 78 & $\begin{array}{l}\text { Matrix inverse can be replaced by } \\
\text { interpolation using a look-up table }\end{array}$ & $\begin{array}{l}\text { Low sensitivity } \\
\text { to noises }\end{array}$ \\
\hline Grid-based & General & Low & $\mathcal{O}\left(n_{g}\right)$ & 9 & $\begin{array}{l}\text { Performance and } n_{g} \\
\text { are not proportional }\end{array}$ & $\begin{array}{l}\text { Low sensitivity } \\
\text { to noises }\end{array}$ \\
\hline
\end{tabular}

application of LPV/robust approaches on autonomous vehicles. In fact, such approaches help to control parameter-variant systems and to handle with environmental disturbances (wind speed, bad road conditions/slopes, etc...).

As future work, a parameter varying actuator model will be investigated to improve the performance. Moreover, a nonlinear bicycle model will be used as a control plant, and the designed controller will be tested in more complex environment (friction drop, gust wind) with higher lateral accelerations.

\section{ACKNOWLEDGMENT}

Authors express their gratitude to the RENAULT research department for its support in developing experimental tests. This paper reflects solely the views of the authors and not necessarily the views of the company they belong to.

\section{REFERENCES}

[1] A. Khodayari, A. Ghaffari, S. Ameli, and J. Flahatgar, "A historical review on lateral and longitudinal control of autonomous vehicle motions," in 2010 International Conference on Mechanical and Electrical Technology, Sep. 2010, pp. 421-429.

[2] F. Navas, V. Milanes, C. Flores, and F. Nashashibi, "Multi-model adaptive control for cacc applications," IEEE Transactions on Intelligent Transportation Systems, 2020.

[3] J. Kosecka, R. Blasi, C. Taylor, and J. Malik, "Vision-based lateral control of vehicles," in Proceedings of Conference on Intelligent Transportation Systems. IEEE, 1997, pp. 900-905.

[4] R. Marino, S. Scalzi, G. Orlando, and M. Netto, "A nested pid steering control for lane keeping in vision based autonomous vehicles," in 2009 American Control Conference, June 2009, pp. 2885-2890.

[5] T.-H. Chang, "Field performance assessment of the advance-f automatic steering control vehicle," Control Engineering Practice, vol. 12, pp. 569576, 052004.

[6] V. Cerone, M. Milanese, and D. Regruto, "Combined automatic lanekeeping and driver's steering through a 2-dof control strategy," IEEE Transactions on Control Systems Technology, vol. 17, pp. 135 - 142, 02 2009.

[7] Hsin-Han Chiang, Li-Shan Ma, Jau-Woei Perng, Bing-Fei Wu, and Tsu-Tian Lee, "Longitudinal and lateral fuzzy control systems design for intelligent vehicles," in 2006 IEEE International Conference on Networking, Sensing and Control, April 2006, pp. 544-549.

[8] S. Chaib, M. S. Netto, and S. Mammar, " $\mathcal{H}_{\infty}$, adaptive, PID and fuzzy control: a comparison of controllers for vehicle lane keeping," in IEEE Intelligent Vehicles Symposium, 2004, June 2004, pp. 139-144.

[9] Z. Lu, B. Shyrokau, B. Boulkroune, S. V. Aalst, and R. Happee, "Performance benchmark of state-of-the-art lateral path-following controllers," 2018 IEEE 15th International Workshop on Advanced Motion Control $(A M C)$, pp. 541-546, 2018.

[10] V. Milanés, D. Llorca, J. Villagra, J. Pérez Rastelli, C. Fernandez, I. Parra, C. González, and M. Sotelo, "Intelligent automatic overtaking system using vision for vehicle detection," Expert Systems with Applications, vol. 39, no. 3, pp. 3362-3373, Feb. 2012. [Online]. Available: https://hal.inria.fr/hal-00741406
[11] C.-F. Lin, J.-C. Juang, and K.-R. Li, "Active collision avoidance system for steering control of autonomous vehicles," IET Intelligent Transport Systems, vol. 8, no. 6, pp. 550-557, 2014.

[12] C. Yu, Y. Zheng, B. Shyrokau, and V. Ivanov, "Mpc-based path following design for automated vehicles with rear wheel steering," in 2021 IEEE International Conference on Mechatronics (ICM). IEEE, 2021, pp. 1-6.

[13] H.-S. Tan and J. Huang, "Design of a high-performance automatic steering controller for bus revenue service based on how drivers steer," Robotics, IEEE Transactions on, vol. 30, pp. 1137-1147, 102014.

[14] S. Schnelle, J. Wang, R. J. Jagacinski, and H.-J. Su, "A feedforward and feedback integrated lateral and longitudinal driver model for personalized advanced driver assistance systems," 2018.

[15] I. Mahtout, F. Navas, D. Gonzalez, V. Milanes, and F. Nashashibi, "Youla-kucera based lateral controller for autonomous vehicle," in 2018 21 st International Conference on Intelligent Transportation Systems (ITSC), Nov 2018, pp. 3281-3286.

[16] M. Safonov, "Origins of robust control: Early history and future speculations," Annual Reviews in Control, vol. 36, p. 173-181, 122012.

[17] D. Leith and W. Leithead, "On formulating nonlinear dynamics in LPV form," in Proceedings of the 39th IEEE Conference on Decision and Control (Cat. No. OOCH37187), vol. 4. IEEE, 2000, pp. 3526-3527.

[18] J. S. Shamma, "Analysis and design of gain scheduled control systems," Ph.D. dissertation, Massachusetts Institute of Technology, 1988.

[19] P. Apkarian and R. J. Adams, "Advanced gain-scheduling techniques for uncertain systems," IEEE Transactions on Control Systems Technology, vol. 6, no. 1, pp. 21-32, Jan 1998.

[20] J. Mohammadpour and C. W. Scherer, Control of linear parameter varying systems with applications. Springer Science \& Business Media, 2012.

[21] O. Sename, P. Gaspar, and J. Bokor (Eds), Robust control and linear parameter varying approaches: application to vehicle dynamics. Springer, LNCIS, 2013, vol. 437.

[22] P. Apkarian, P. Gahinet, and G. Becker, "Self-scheduled $\mathcal{H}_{\infty}$ control of linear parameter-varying systems: a design example," Automatica, vol. 31, no. 9, pp. 1251-1261, 1995.

[23] G. Z. Angelis, "System analysis, modelling and control with polytopic linear models." 2003.

[24] F. Wu, "Control of linear parameter varying systems," Ph.D. dissertation, University of California at Berkeley, 1995.

[25] P. Apkarian and P. Gahinet, "A convex characterization of gainscheduled $H_{\infty}$ controllers," IEEE Transactions on Automatic Control, vol. 40, no. 5, pp. 853-864, May 1995.

[26] C. Hoffmann and H. Werner, "A survey of linear parameter-varying control applications validated by experiments or high-fidelity simulations," IEEE Transactions on Control Systems Technology, vol. 23, no. 2, pp. 416-433, March 2015.

[27] E. Alcala, V. Puig, J. Quevedo, and T. Escobet, "Gain-scheduling lpv control for autonomous vehicles including friction force estimation and compensation mechanism," IET Control Theory \& Applications, vol. 12, no. 12 , pp. $1683-1693,2018$

[28] J. Guo, Y. Luo, and K. Li, "Robust gain-scheduling automatic steering control of unmanned ground vehicles under velocity-varying motion," Vehicle System Dynamics, pp. 1-22, 052018.

[29] V. T. Vu, O. Sename, L. Dugard, and P. Gáspár, "The design of an $\mathcal{H}_{\infty} / \mathrm{LPV}$ active braking control to improve vehicle roll stability," in 7th IFAC Symposium on Systems Structure and Control (SSSC 2019), Sinaia, Romania, Sep. 2019. [Online]. Available: https://hal.archives-ouvertes.fr/hal-02156867

[30] K. Yamamoto, O. Sename, D. Koenig, and P. Moulaire, "Design and experimentation of an LPV extended state feedback control on electric power steering systems," Control Engineering Practice, vol. 90, pp. 123$132,2019$. 
[31] C. Poussot-Vassal and C. Roos, "Generation of a reduced-order LPV/LFT model from a set of large-scale MIMO LTI flexible aircraft models," Control Engineering Practice, vol. 20, no. 9, pp. 919-930, 2012.

[32] J.-M. Biannic, C. Roos, and A. Knauf, "Design and robustness analysis of fighter aircraft flight control laws," European journal of control, vol. 12, no. 1, pp. 71-85, 2006.

[33] E. Roche, O. Sename, and D. Simon, "LFT/H $\mathcal{H}_{\infty}$ varying sampling control for autonomous underwater vehicles," IFAC Symposium on System, Structure and Control, 2010.

[34] C. Gauthier, O. Sename, L. Dugard, and G. Meissonnier, "Some experimental results of an $\mathcal{H}_{\infty}$-LPV controller applied to a diesel engine common rail injection system," IFAC Proceedings Volumes, vol. 40, no. 10, pp. 417-424, 2007.

[35] H. Pacejka, Tire and vehicle dynamics. Elsevier, 2005.

[36] R. Rajamani, Vehicle dynamics and control. New York, USA: Springer Science \& Business Media, 2011. [Online]. Available: http://standards.sae.org/j3016201401

[37] F. Gottmann, H. Wind, and O. Sawodny, "On the influence of rear axle steering and modeling depth on a model based racing line generation for autonomous racing," in 2018 IEEE Conference on Control Technology and Applications (CCTA), 2018, pp. 846-852.

[38] C. Hoffmann and H. Werner, "Complexity of implementation and synthesis in linear parameter-varying control," IFAC Proceedings Volumes, vol. 47, no. 3, pp. 11749-11760, 2014.

[39] D. Robert, O. Sename, and D. Simon, "A reduced polytopic LPV synthesis for a sampling varying controller: Experimentation with a inverted pendulum," in 2007 European Control Conference (ECC), July 2007, pp. 4316-4323.

[40] C. Briat, Linear Parameter-Varying and Time-Delay Systems. Analysis, Observation, Filtering Control. Springer-Verlag, 2015.

[41] S. Boyd, L. El Ghaoui, E. Feron, and V. Balakrishnan, Linear matrix inequalities in system and control theory. Siam, 1994, vol. 15.

[42] K. Berntorp and S. Di Cairano, "Tire-stiffness and vehicle-state estimation based on noise-adaptive particle filtering," IEEE Transactions on Control Systems Technology, vol. 27, no. 3, pp. 1100-1114, 2019.

[43] W. Wang, J. Doyle, C. Beck, and K. Glover, "Model reduction of lft systems," in [1991] Proceedings of the 30th IEEE Conference on Decision and Control, Dec 1991, pp. 1233-1238 vol.2.

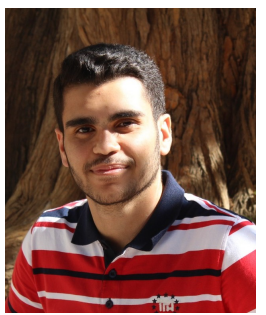

Hussam Atoui received his B.Sc. degree in Mechanical Engineering from Lebanese University, Beirut, Lebanon in 2019 and the M.Sc. degree in Automatic Control from Grenoble-Alpes University, Grenoble, France, in 2019. He is currently working toward the Ph.D. degree with Grenoble-Alpes University in a joint research between Gipsa-lab and Renault. His research interests concern switching control, robust control, and mainly control and motion planning of autonomous vehicles.

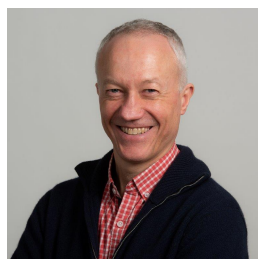

Olivier Sename received a Ph.D. degree from Ecole Centrale Nantes in 1994. He is now Professor at the Institut Polytechnique de Grenoble within GIPSAlab. His main research interests include Linear Parameter Varying systems and automotive applications. $\mathrm{He}$ is the (co-)author of 2 books, 60 international journal papers, and more than 200 international conference papers. He was the General Chair of the IFAC Joint Conference SSSC-TDSFDA 2013, of the 1st IFAC Workshop on Linear Parameter Varying Systems 2015 and he was the IPC Chair of the 2nd IFAC Workshop LPVS 2018. He has led several industrial (Renault, Volvo Trucks, JTEKT, Delphi) and international (Mexico, Italy, Hungary) collaboration projects. He has supervised 32 Ph.D. students.

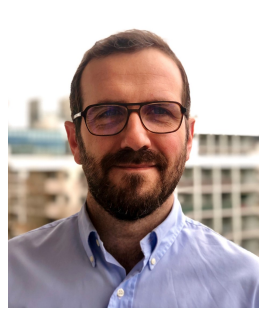

Vicente Milanés received his Ph.D. degree in electronic engineering from University of Alcalá, Madrid, Spain, in 2010. He was with the AUTOPIA program at the Center for Automation and Robotics (UPM-CSIC, Spain) from 2006 to 2011. Then, he was awarded with a two-years Fulbright fellowship at California PATH, UC Berkeley. In 2014, he joined the RITS team at INRIA, France. Since 2016, he is with the Research Department at Renault, France. $\mathrm{He}$ is the author or a coauthor of more than 120 refereed publications in international journals, book chapters, and conference proceedings; and more than 10 industrial patents. His research interests cover multiple aspects in the autonomous vehicle field.

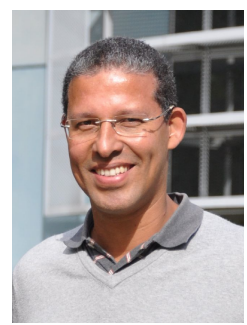

John J. Martinez was born in Cali, Colombia. He received the B.Sc. degree in electrical engineering and the M.Sc. degree in automatic control from the Universidad del Valle, Cali, in 1997 and 2000, respectively, and the Ph.D. degree in automatic control from the Institut National Polytechnique de Grenoble, Grenoble, France, in 2005. He joined the Universidad Nacional de Colombia, Medellin, Colombia, as a Teacher Assistant, from 2001 to 2002. He was an Invited Visitor with the Centre for Complex Dynamic Systems and Control, The Newcastle University, Callaghan, NSW, Australia, in 2005, 2007, and 2009. He is currently an associate professor at Grenoble-INP and researcher at GIPSA-lab (Control System Department). His research interest is related to modeling and robust control of mechatronic systems (e.g. Polytopic system modeling, Linear Parameter-Varying systems, Switching control and Invariant-Set Theory for Fault-Tolerant Control and Robust Disturbance Estimation/Rejection), mostly in the following applications: Automotive vehicle dynamics Safety, Aerial vehicle dynamics, Wind turbines control, Physiologic-aware electric bikes and Anti-vibration systems. 